\title{
Esophagobronchial Fistula Complicating Isolated Esophageal Crohn's: A Case Report
}

\author{
Armand Asarian ${ }^{\mathrm{a}}$, Olubunmi Esan ${ }^{\mathrm{a}}$, Segun Adeoye ${ }^{\mathrm{b}, \mathrm{c}}$
}

\begin{abstract}
Isolated esophageal Crohn's is a rare condition with few reported cases; there are even much fewer documented case reports of esophagobronchial fistula complicating esophageal Crohn's. We present a case of isolated esophageal Crohn's with esophagobronchial fistula presenting with hemoptysis. Diagnostic considerations and therapeutic options are detailed. We recommend chest computerized tomography (with computerized virtualization of endoluminal data when available) as the imaging modality of choice, as well as traditional studies: barium swallow, bronchoscopy and upper gastrointestinal endoscopy, for a complete elucidation of all associated clinical entities. We conclude by making the case for entertaining a diagnosis of esophagobronchial or related fistulas in patients with Crohn's disease who present with interval development of respiratory symptoms or signs especially when these are persistent or recurrent.
\end{abstract}

Keywords: Esophageal Crohn's; Esophagobronchial fistula; Hemoptysis

\section{Introduction}

Crohn's disease (CD) is an idiopathic chronic inflammatory bowel disease characterized pathologically by the development of chronic segmental mucosal and transmural inflammation, granulomata and fistulae with adjacent organs. Clinically, CD presents with a myriad of constitutional, systemic and gastrointestinal symptoms and signs. Although any part

\footnotetext{
Manuscript accepted for publication November 22, 2013

${ }^{a}$ Department of Surgery, The Brooklyn Hospital Center, Brooklyn, NY, USA

${ }^{\mathrm{b}}$ University of Pittsburgh Medical Center (UPMC), Horizon, Greenville, PA, USA

${ }^{\mathrm{c}}$ Corresponding author: Segun Adeoye, University of Pittsburgh

Medical Center (UPMC), Horizon, Greenville, PA, USA.

Email: adeoyesp@upmc.edu
}

doi: http://dx.doi.org/10.14740/jmc1601w of the alimentary canal may be affected, $\mathrm{CD}$ has a great predilection for the ileum, colon and the anorectal regions of the alimentary tract. CD involving the esophagus is uncommon [1].

There are few reported cases of isolated esophageal CD (probably under-reported) [1-3], and even much fewer reported cases of esophageal CD with esophagobronchial fistula. The estimated incidence of esophageal CD is $0.3-2 \%$ and affects as much as $6.5 \%$ of the adult and pediatric population with $\mathrm{CD}[1,4,5]$. Brittenden and colleagues in Leeds reported a case of cough and recurrent pneumonias complicating esophagobronchial fistula in a patient with CD [6]. Dyer et al are among the few who have documented cases of esophageal CD complicated by fistula with the respiratory tract $[7,8]$.

The history is often that of interval development of respiratory symptoms or signs (coughing upon eating, hemoptysis, recurrent pneumonias) in the setting of pre-diagnosed esophageal or gastrointestinal CD. The diagnosis demands combinations of barium swallow, chest-CT, and endoscopies of respiratory and upper gastrointestinal tract, as well as histopathologic confirmation of biopsied tissue from these endoscopic studies. Intervention is necessary to prevent pneumonic, and hemorrhagic complications. Treatment of lower esophageal Crohn's complicated by esophagobronchial fistula includes definitive surgery: esophago-gastrectomy and cervical esophagostomy with interval immunomodulatory therapy to control synchronous and metachronous esophageal, gastrointestinal and extra-intestinal disease. We present a case of isolated esophageal Crohn's complicated by the development of esophagobronchial fistula.

\section{Case Report}

$\mathrm{AB}$, a 30-year-old Asian male presented to the ER with hematemesis and hemoptysis of 2 days duration. Medical history was significant for 10-year history of esophageal CD in remission for the past 6 years. The patient was not taking any medication. He reported the absence of fever, night sweats, chills or recent weight loss. There was no recent or remote smoking history. On physical examination, he appeared 


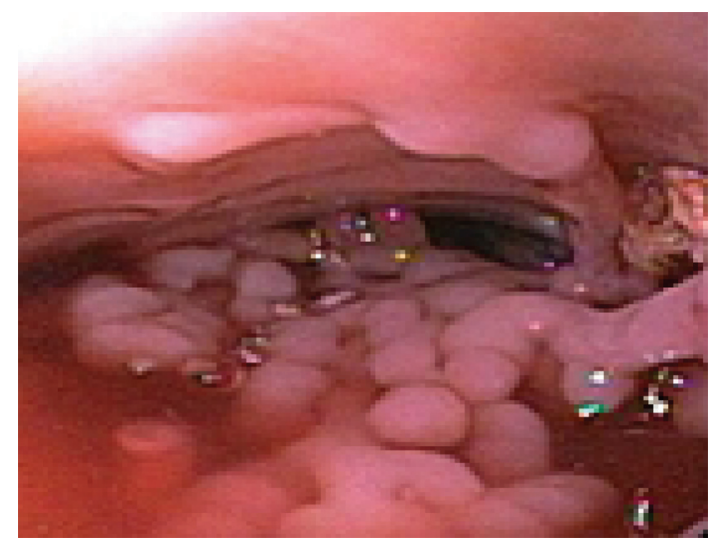

Figure 1. Esophagoscopy showing lower esophageal pseudopolyps and minimal scarring.

malnourished with significant muscle atrophy. The remainder of the examination including abdominopelvic palpation and chest auscultation was unremarkable. Laboratory studies reported a hemoglobin level of $12 \mathrm{~g} / \mathrm{dL}, \mathrm{WBC} 6,400 / \mathrm{dL}$, platelet count 500,000/dL, an erythrocyte sedimentation rate (ESR) of $40 \mathrm{~mm} / \mathrm{h}$ and C-reactive protein of $5 \mathrm{mg} / \mathrm{dL}$. Serologic markers for hepatitis and human immunodeficiency viruses (HIV) were negative, as was a tuberculin skin test. Chest X-ray was normal.

Upper endoscopy revealed multiple friable, confluent ulcers with pseudopolyposis and granulation tissue in the mid-esophagus without evidence of active bleeding (Fig. 1). Fiberoptic bronchoscopy showed normal appearing endoluminal mucosa with fresh blood in the left main bronchus and a localized bleeding site. Chest-CT with and without intravenous contrast showed residual area of consolidation in the left para-aortic region and air seen in the periphery of the esophagus adjacent to trachea and left bronchus, with minimal bronchial wall thickening suspicious for esophagobronchial/trachea fistula. As patient's history, chest-CT and endoscopic studies were nearly conclusive, a barium swallow study was not conducted. Intra-operatively, the patient was found to have an esophagobronchial fistulous tract extending into left main stem bronchus. An esophago-gastrectomy with cervical esophagostomy was performed. The biopsy of the tract showed mucosal bridging, filiform polyposis and focal transmural scarring consistent with $\mathrm{CD}$ at esophagobronchial fistula site.

The patient did well post-operatively and was discharged to follow-up with the gastroenterology and cardiothoracic surgery units, with plan to institute medical immunomodulatory therapy.

\section{Discussion}

In the case presented above, we were not burdened with the arduous task of making the initial diagnosis of isolated esophageal $\mathrm{CD}$ as the patient had the diagnosis at presentation. A description of the chronology of events preceding the admitting diagnosis is outside the scope and intent of this paper. The diagnosis of an isolated esophageal CD is often a sequel of endoscopy and histopathology of biopsied tissue done for work-up of upper gastrointestinal symptomatology or in patient with extra-intestinal features of CD [5]. In certain circumstances, absent the extra-intestinal manifestations of $\mathrm{CD}$, an incidental endoscopic finding results when investigating a patient for usual symptoms relatable to the upper gastrointestinal tract [5]. Differentiating isolated mid-lower esophageal CD clinically from more common differentials like functional dyspepsia, peptic ulcer disease, GERD and gastroesophageal hernia is difficult [2]. Histopathology, imaging and occasionally, serology and microbiologic techniques may be needed to clinch a definitive diagnosis or at least exclude some differential diagnoses.

The case demonstrates the potential for extension of the inflammatory process associated with $\mathrm{CD}$ to, and the development of fistulous connections with visceral and body structures adjacent to the esophagus. Reider and colleagues allude to this potential complication in the course of CD [9]. $\mathrm{CD}$ almost exclusively affects the distal two-thirds of the esophagus (with only one reported case of the isolated upperthird involvement) [2]. The relation of the distal two-thirds of the esophagus to the tracheobronchial tree and lungs, the pericardium and the left atrium anteriorly, its surrounding structures in the inferior mediastinum and the respiratory diaphragm inferiorly create the potential for contiguous extension of esophageal inflammation and the development of fistulous connections.

The gravity of inflammatory extensions ranges from clinical trivialities like subclinical para-esophageal inflammation to catastrophic presentations like pan-mediastinopleuropericarditis, esophageal perforation and fistula formation (with severe recurring aspiration and pneumonias) and life-threatening hemorrhage. It sounds logical to infer that the much less common esophageal involvement in $\mathrm{CD}$ and the effectiveness of current medical therapies may explain the rarity of these complications. The differences in thoracic and abdominal anatomy make the potential for development of life-threatening complications more likely with esophageal disease (than with intestinal disease), were the incidence of esophageal and gastrointestinal CD similar.

The complaint of hemoptysis, the finding of fresh blood in the left bronchus on bronchoscopy and the CT-chest report demonstrating extraluminal air and para-aortic/paraesophageal consolidation are consistent with the presence of esophageal perforation and/or esophagobronchial/tracheal fistula. These findings are comparable to radiologic findings by Chaky et al (2008) in their report on chest-CT diagnosis of adult bronchoesophageal fistula [10] and Erasmus et al (2002) report on esophageal perforation [11]. Chaky et al 
(2008) also report that the fistula orifice can be demonstrated by a virtual endoluminal view using CT data [10]. We recommend chest-CT with and without contrast (and virtualization of endoluminal data when available) as the imaging modality of choice. Bronchoscopy may be normal or show varying degrees of endoluminal erythema and thickening, with or without obstruction or fistula [10]. The closer relation of the left bronchus to the esophagus compared with other parts of the tracheobronchial tree readily explains the more likely incidence of left broncho-esophageal fistula.

In this case, the short history of hemoptysis (2 days), the absence of altered blood on bronchoscopy and a relatively high hemoglobin concentration suggest a recent completion of the fistulous process. The fact that the patient had not been taking medication for $\mathrm{CD}$, assessment of malnourishment on physical examination and the elevated ESR suggests a chronic, indolent disease process. Furthermore, the presence of para-aortic consolidation (without sympathetic left pleural effusion) and para-esophageal air (mediastinal emphysema), the absence of lung consolidation and the lack of leucocytosis lays credence to the hypothesis that the extension of the transmural esophageal inflammation into the adjacent inferior mediastinum was a recent rather than a remote event. The chronicity of the inflammatory response in CD notwithstanding, leucocytosis may complicate the picture especially when organic esophageal products leak into the mediastinum or pleural cavity, eliciting a severe inflammatory response. In a few patients, chronicity of respiratory symptoms like coughing, dyspnea, inspiratory crackles or expiratory wheezing may necessitate pulmonologist referral and pulmonary function testing.

The initial hemoglobin and hematocrit may not reflect the amount of internal hemorrhage. Serial hemoglobin measurement to accurately assess extravascular loss is required. The absence of leucocytosis is not unexpected considering the chronicity of the inflammation and the tendency to walloff the inflammatory response in CD. More so, leucocytosis may not be evident in patients with chronic disease who are malnourished and/or taking anti-inflammatory and immunosuppressant/modulators. In these cases, a thorough clinical examination, serologic inflammatory markers and imaging studies hold the key to the complete elucidation of all clinical entities at play.

We hypothesize that the most probable chronology of events in this case is the subclinical transmural extension of mid-third esophageal disease into the inferior mediastinum with subsequent metachronous fistula tract development into the left bronchus. Erosion into the left bronchus resulted in bronchial hemorrhage and hemoptysis, heralding the development of the fistula. We reason that the fistulous process involved a non-major branch of the left bronchial artery. The early presentation and apparent sparing of major bronchial vessels may explain the patient's relatively benign presentation and the uneventful outcome. A more ominous presenta- tion is severe anemia from torrential bronchial hemorrhage, and severe respiratory distress from pulmonary hemorrhage, chemical or septic pneumonitis, empyema, pleural effusion and pulmonary edema. The case presented supports entertaining the diagnosis of esophagobronchial and related fistulas, especially in the setting of gastrointestinal and/or extraintestinal manifestation of $\mathrm{CD}$ with persistent or recurring respiratory symptoms.

The incident bronchial hemorrhage and the potential complications of esophagobronchial fistula made emergency esophago-gastrectomy with cervical esophagostomy the treatment modality of choice, even when post-operative recurrences are common. In view of all the clinical entities present in this case, the attendant urgency and the resources available at our facility, it is our opinion that medical immunomodulating therapies, and interventional radiology modalities like fistula closures using artificial sealants [8] and bronchial artery embolization are either inappropriate or therapeutically inferior to surgical intervention. The pathology of esophageal CD related esophagobronchial fistula makes staple-ligation of fistula as well as other closure technique for acquired broncho-esophageal fistula non-viable surgical options.

\section{Conclusion}

This case leads us to conclude that in the natural course of $\mathrm{CD}$, the potential for inflammation and fistulous connections to gastrointestinal tract is the same for adjacent organs above and below the respiratory diaphragm. Unexplained respiratory symptomatology in a patient CD should cause the consideration of esophagobronchial and related fistulas and necessitate appropriate investigation.

\section{Competing Interest and Financial Disclosures}

The paper received no form of funding or financial support from any public or private organization. The authors have no conflict of interest issues to declare.

\section{References}

1. Feagans J, Victor D, Joshi V. Crohn disease of the esophagus: a review of the literature. South Med J. 2008;101(9):927-930.

2. Crohn, B, Ginzburg, L, Oppenheimer, G. Regional ileitis: a pathologic and clinical entity. JAMA. 1932;99:13231329.

3. Zezos P, Kouklakis G, Oikonomou A, Pitiakoudis M, Simopoulos C. Esophageal Crohn's disease treated "topically" with swallowed aerosolized budesonide. Case Rep Med. 2010;2010. 
4. Geboes K, Janssens J, Rutgeerts P, Vantrappen G. Crohn's disease of the esophagus. J Clin Gastroenterol. 1986;8(1):31-37.

5. Lopez-Rubio F, Naranjo-Rodriguez A., Solorzano-Peck G. Isolated esophageal involvement of Crohn's disease. Eur J Gastroenterol Hepatol. 2003;15:1123-1126.

6. Brittenden J, Hosker HS, Healey C, Porter G. Oesophago-bronchial fistula causing cough and recurrent lower respiratory tract infections in a patient with Crohn's disease. Hosp Med. 2005;66(5):310-311.

7. Steel A, Dyer NH, Matthews HR. Cervical Crohn's disease with oesophago-pulmonary fistula. Postgrad Med J. 1988;64(755):706-709.

8. Stiewe S, Nitzsche H. [Crohn disease of the esoph- agus with esophageal-pulmonary fistula]. Rofo. 1998;169(5):562-563.

9. Rieder F, Hamer O, Gelbmann C, Scholmerich J, Gross V, Feuerbach S, Herfarth H, et al. Crohn's disease of the esophagus: treatment of an esophagobronchial fistula with the novel liquid embolic polymer "onyx". Z Gastroenterol. 2006;44(7):599-602.

10. Chaky, D, Escamilla, C, Sheridan, P, Deboer, D. Adult bronchoesophageal fistula diagnosed on computed tomography. Radiology Case Reports. 2008;3(2). DOI: 10.2484/rcr.2008.v3i2.126.

11. Gimenez A, Franquet T, Erasmus JJ, Martinez S, Estrada P. Thoracic complications of esophageal disorders. Radiographics. 2002;22(Spec No):S247-258. 